\title{
BMJ Open Lifestyle Intervention in Chronic Ischaemic Heart Disease and Type 2 Diabetes (the LeIKD study): study protocol of a prospective, multicentre, randomised, controlled trial
}

Pia von Korn (1) , ${ }^{1,2}$ Hanna Sydow, ${ }^{3,4}$ Sarah Neubauer, ${ }^{5}$ André Duvinage, ${ }^{1,2}$
Anja Mocek, ${ }^{3}$ Sophia Dinges, ${ }^{1}$ Bjoern Hackenberg, ${ }^{6}$ Mario Weichenberger, ${ }^{1}$
Julia Schoenfeld, ${ }^{1,2}$ Volker Amelung, ${ }^{3,4}$ Stephan Mueller, ${ }^{1,2}$ Martin Halle ${ }^{1,2}$

To cite: von Korn P, Sydow H, Neubauer S, et al. Lifestyle Intervention in Chronic Ischaemic Heart Disease and Type 2 Diabetes (the LelKD study): study protocol of a prospective, multicentre, randomised, controlled trial. BMJ Open 2021;11:e042818. doi:10.1136/ bmjopen-2020-042818

- Prepublication history and additional materials for this paper is available online. To view these files, please visit the journal online (http://dx.doi. org/10.1136/bmjopen-2020042818).

SM and MH are joint senior authors.

Received 15 July 2020

Revised 18 January 2021

Accepted 25 January 2021

Check for updates

(C) Author(s) (or their employer(s)) 2021. Re-use permitted under CC BY-NC. No commercial re-use. See rights and permissions. Published by BMJ.

For numbered affiliations see end of article.

Correspondence to Professor Martin Halle; Martin.Halle@mri.tum.de

\section{ABSTRACT}

Introduction Guidelines recommend lifestyle intervention in chronic ischaemic heart disease (CIHD) and type 2 diabetes mellitus (T2DM). However, evidence from randomised controlled trials is scarce in patients with combined entities.

Methods and analysis The Lifestyle Intervention in Chronic Ischaemic Heart Disease and Type 2 Diabetes (LelKD) trial is a prospective, multicentre study that will randomise (1:1) patients with CIHD (ICD-10: I20-I25) and T2DM (ICD-10: E11) from one health insurance company into a lifestyle intervention (LS) or usual care (UC). Active LS consists of an individual combined exercise programme of strength and endurance training and nutritional counselling with regular feedback for 6 months. Intervention is supported by telemedicine. Follow-up without individualised feedback will continue for 6 months. The study aims to investigate whether an individualised telemedical supported LS intervention is superior to UC in improving cardiovascular risk factors, physical activity, quality of life, health literacy, major cardiovascular events and health economics in patients with both $\mathrm{CIHD}$ and T2DM. Primary endpoint is the change in $\mathrm{HbA}_{1 \mathrm{c}}$ from baseline to 6 months.

Ethics and dissemination The study has been approved by the ethics committee of the Technical University of Munich (registration number: 144/18-S) and at each study site. The study will be conducted according to the World Medical Association Declaration of Helsinki, and results will be published in articles and reports. It is funded by the Federal Joint Committee (www.innovationsfonds.g-ba.de), reference number 01NVF17015, which has no impact on data collection, analysis or interpretation. Dissemination is independent of the funding source.

Trial registration number Clinical trials.gov identifier: NCT03835923. German registry for clinical studies (DRKS): DRKS00015140.

\section{INTRODUCTION}

Patients with both chronic ischaemic heart disease (CIHD) and type 2 diabetes mellitus

\section{Strengths and limitations of this study}

- The LelKD (Lifestyle Intervention in Chronic Ischaemic Heart Disease and Type 2 Diabetes) study is one of the largest randomised controlled trials investigating a telemedically supported lifestyle intervention in patients suffering from both chronic ischaemic heart disease and type 2 diabetes mellitus.

- This study evaluates an approach for both patients and healthcare providers with regard to a telemedically supported lifestyle intervention integrating exercise and nutrition in a multimorbid elderly population.

- This study combines health economic data from one health insurance company with telemedical data from individualised exercise and nutrition.

- This study is limited to patients willing to use smartphones.

- This study is limited to insurants from one health insurance company, which may limit generalisation.

(T2DM) have a high morbidity and mortality. ${ }^{1}$ Results from the EUROASPIRE-IV survey $(n=7998)$, assessing the implementation of current guidelines in secondary prevention, show that $26.8 \%$ of patients with CIHD also suffer from T2DM. ${ }^{2}$ Combining the diagnosis of T2DM and CIHD exponentially increases the risk of impaired quality of life (QoL) and mortality. ${ }^{13}$

Recommendations for lifestyle intervention are included in current guidelines in CIHD $^{1}$ and T2DM. ${ }^{4}$ Both have received I A classifications, which is based on evidence from randomised controlled intervention trials. The largest trial (Look AHEAD, n=5145) in T2DM ( $14 \%$ with former cardiovascular event) has revealed that lifestyle intervention 
including exercise training in combination with a hypocaloric diet improved glycosylated haemoglobin $\left(\mathrm{HbA}_{1 \mathrm{c}}\right)$ over 1 year as compared with usual care. ${ }^{5}$ Moreover, results from the ENHANCE trial in patients with T2DM (1:1 randomised, $\mathrm{n}=296$, behavioural intervention, increasing diabetes self-care by monitoring software) have shown a trend towards a mean reduction of $\mathrm{HbA}_{1 \mathrm{c}}$ of $0.4 \%$ after 6 months, although this was non-significant due to improvements in both experimental and control groups. ${ }^{6}$ Furthermore, results of the DiRECT study $(n=306)$ in an outpatient general practitioner setting have demonstrated a $46 \%$ remission of T2DM through weight reduction by meal replacement therapy and exercise counselling after 12-month intervention. ${ }^{7}$

Evidence from a recent study in patients with stable CIHD ( $\mathrm{n}=15487$, physical activity (PA) data by questionnaire, $38.7 \%$ T2DM) has revealed that habitual exercise is significantly associated with lower cardiovascular mortality (adjusted $\mathrm{HR}=0.92) .{ }^{8}$ Moreover, in patients with heart failure with reduced ejection fraction (HFrEF), the HF-ACTION study ( $\mathrm{n}=2331,51.4 \%$ ischaemic aetiology of heart failure, prevalence of T2DM 32.1\%) has revealed that, after adjusting for prognostic factors, exercise training significantly reduced rehospitalisation rate (HR 0.85, $\mathrm{p}=0.03){ }^{9}$

However, in all of these trials, adherence to intervention has been the key challenge. ${ }^{59}$ Telemedicine applications, characterised by the WHO as any information and communication technologies that allow remote healthcare services, ${ }^{10}$ may support implementation of and adherence to lifestyle measures, as demonstrated by the ENHANCE trial. ${ }^{6}$ A recent review by Zhu et al (2019) has underlined the effectiveness of telemedical interventions (defined by telephone, vocal support or telemonitoring) in patients with heart failure (10981 patients with HFrEF, NYHA I-IV, 29 randomised controlled trials) by revealing significant reductions in hospitalisation rate, all-cause mortality and length of hospitalisation in the intervention group. ${ }^{11}$ The TIM-HF2 trial investigated the efficacy of a remote patient management (including medical multicomponent telemonitoring system with daily transmission to study centre, monthly phone calls/interviews and option of emergency phone contact) in $\mathrm{n}=1571$ patients with heart failure (New York Heart Association (NYHA) II-III, 1:1 randomisation) on morbidity and mortality $(40 \% \text { ischaemic cause of heart failure, } 45 \% \mathrm{~T} 2 \mathrm{DM})^{12}$ and revealed a significant difference in days lost to cardiovascular hospital admissions (4.9\% in remote patient management vs $6.6 \%$ in $\mathrm{UC}, \mathrm{p}=0.046$ ).

\section{Rationale}

Despite this current positive clinical evidence of lifestyle interventions in T2DM and CIHD, the challenge of implementation and adherence to a long-term lifestyle programme remains unresolved. To overcome these major challenges, telemedicine has revealed beneficial effects on adherence and motivation by improving

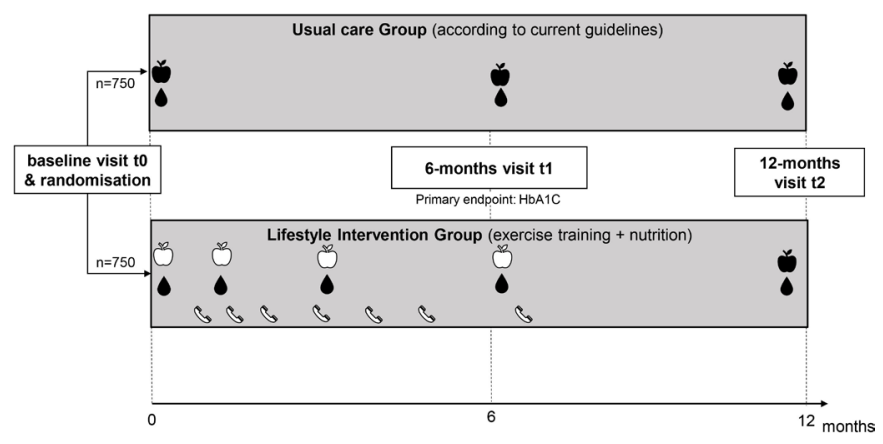

Figure 1 LelKD (Lifestyle Intervention in Chronic Ischaemic Heart Disease and Type 2 Diabetes) study design. Apple black: food diary without feedback; apple white: food diary with feedback; blood drop: blood glucose profile without feedback; phone: feedback training from core laboratory.

patients' education and awareness ${ }^{12}$; however, this approach is unclear in an elderly and multimorbid patient population.

Therefore, a large, randomised controlled trial assessing the combined effects of exercise and nutrition supported by telemedicine in patients suffering from CIHD and T2DM has yet to be conducted. Data from health economics will be particularly valuable for subsequent implementation into standard care programmes in this population.

\section{METHODS AND ANALYSIS \\ Study objectives}

We hypothesise that patients with CIHD and T2DM receiving a telemedical lifestyle intervention programme including exercise training and nutritional advice will show significant improvements in cardiovascular risk factors, PA, health literacy, health economic data and clinical events when assessed after 6 and 12 months, as compared with patients assigned to UC.

\section{Study design}

The LeIKD study is a prospective, multicentre, randomised, controlled trial. Figure 1 depicts the study flow chart for further details. We used the SPIRIT (Standard Protocol Items: Recommendations for Interventional Trials) checklist when writing our report. ${ }^{13}$ The study will include patients with the diagnosis of both CIHD and T2DM. Patients will be identified by the statutory health insurance fund TK (Techniker Krankenkasse, Hamburg, Germany) according to ICD (International Classification of Diseases) codes for ischaemic heart diseases (ICD-10: I20-I25, ie, history of angina pectoris; acute myocardial infarction; subsequent ST elevation (STEMI) and non-ST elevation (NSTEMI) myocardial infarction; certain current complication following ST elevation (STEMI) and non-ST elevation (NSTEMI) myocardial infarction; other acute ischaemic heart diseases; CIHD) and T2DM (ICD-10: E11). A complete list of inclusion and exclusion criteria is shown in table 1. 
Table 1 Inclusion and exclusion criteria

\begin{tabular}{|c|c|}
\hline Inclusion criteria & Exclusion criteria \\
\hline $\begin{array}{l}\text { Ischaemic heart disease } \\
\text { (ICD-10: I20-I25) } \\
\text { Diabetes mellitus } \\
\text { (ICD-10: } E 11 \text { ) and } \\
\text { HbA1c } \geq 6.5 \text { or anti- } \\
\text { diabetic medication at } \\
\text { the time of screening }\end{array}$ & $\begin{array}{l}\text { Mental and behavioural disorders } \\
\text { (ICD-10: F00, F01, F02, F11, F12, } \\
\text { F13, F14, F15, F16, F18, F20, F21, } \\
\text { F22, F23, F24, F25, F28, F29, F44, } \\
\text { F72, F73, F17, F84) }\end{array}$ \\
\hline$\geq 18$ years & Heart failure NYHA IV (ICD-10: I50.14) \\
\hline $\begin{array}{l}\text { Insured at TK health } \\
\text { insurance fund }\end{array}$ & $\begin{array}{l}\text { Malignant neoplasm (ICD-10: C25, } \\
\text { C34, C56, C72, C73, C78, C79, C97) }\end{array}$ \\
\hline $\begin{array}{l}\text { Permission to do } \\
\text { physical exercises by } \\
\text { study investigator }\end{array}$ & Parkinson's disease (ICD-10: G20) \\
\hline
\end{tabular}

Written informed consent Alzheimer disease (ICD-10: G30) Infantile cerebral palsy (ICD-10: G80)

Chronic kidney disease (ICD-10: N18.4 \& N18.5)

Trisomy 21 (ICD-10: Q90)

Blindness/visual impairment (ICD-10: H54.0, H54.2, H54.3)

Hearing loss (ICD-10: H90.0, H90.3, H90.5, H90.6, H90.8)

\section{Care level 1-5}

Insured abroad

Inability to do physical exercises or conditions that may interfere with exercise intervention

No optimal cardiac treatment within the last 4 weeks to be assessed and decided on by the local investigator

Not clinically stable within the last 4 weeks to be assessed and decided on by the local investigator

Participation in another trial

Care level: needed degree of required assistance in daily life (part of the German health system).

ICD, International Classification of Diseases register; NYHA, New York Heart Association.

Eleven centres across Germany have been selected as certified study sites: Aachen (University Hospital, Department for Cardiology), Berlin (Charité, Department for Sports Medicine and Department for Cardiovascular Prevention and Heart Failure), Dresden (University Hospital, Department for Internal Medicine and Cardiology), Freiburg (University Hospital, Department for Cardiology and Angiology), Greifswald (University Hospital, Department for Internal Medicine), Kassel (private practice for Cardiology), Leipzig (University Hospital, Department for Cardiology), Magdeburg (University Hospital, Department for Cardiology and Angiology), Villingen-Schwenningen (private practice for Prevention and Therapy) and the Department for Prevention and Sports Medicine at University Hospital
'Klinikum rechts der Isar' of the Technical University of Munich. Target recruitment rates are derived by the number of insurants at Techniker Krankenkasse with the corresponding ICD diagnoses. Standardised data collection and administration in each site is assured by standard operating procedures (SOPs) and personal training by the trial committee before inclusion of the first patient per site. When living within the area of $50 \mathrm{~km}$ from a site, patients will be contacted via phone by the health insurance fund and informed on the background and purpose of the study. In case of consent to participate in the study, the contact details of the potential study participant will be consensually transmitted to the local study site via pincode safe data room to schedule a screening visit (t0).

\section{Randomisation}

All patients will be randomly assigned to either the LS or UC by means of block randomisation, stratified by centre, using a web-based electronic data capture system (secuTrial, interActive Systems GmbH, Berlin, Germany).

\section{Baseline and follow-up investigations}

After written informed consent, anamnesis including medical history, medication, physical examination, anthropometry and resting ECG will be performed. Blood samples will be taken and analysed for standard laboratory values $\left(\mathrm{HbA}_{1 c}\right.$, blood count, blood lipids, N-terminal pro-brain natriuretic peptide) in a local laboratory.

Health literacy, eating behaviour, daily PA and QoL will be assessed by the European Health Literacy Questionnaire (HLS-EU-Q16), a German questionnaire on eating behaviour ('Fragebogen zum Essverhalten', FEV), the International Physical Activity Questionnaire (IPAQ) and the Short Form Health Survey (SF-36), respectively. These examinations will be repeated after $6(\mathrm{t} 1)$ and 12 months (t2).

Furthermore, a symptom-limited maximal cardiopulmonary exercise test (CPET) on a stationary cycle ergometer will be performed according to current recommendations at baseline and after 6 months. ${ }^{14}$ An exercise stress test (including exercise ECG) will be performed after 12 months. Breath-by-breath CPET data will be transferred to and analysed by the CPET core laboratory at Technical University of Munich (TUM).

To monitor daily PA and blood glucose levels, all patients (both groups) will receive a pedometer (AS80/ AS87, Beurer GmbH, Ulm, Germany) and blood glucose metre (GL50evo, Beurer GmbH, Ulm, Germany), which are connected to an app (Beurer Health manager, Beurer GmbH, Ulm, Germany). While the pedometer should be worn throughout the entire duration of the study, patients are asked to measure 3-day blood glucose profiles at three different points in the study: during week 1 (after $t 0$ ), the week after $\mathrm{t} 1$ and the week before $\mathrm{t} 2$. Randomised patients are equipped with relevant smartphone applications (apps). If necessary, a smartphone will be provided free of cost for the duration of the study. To ensure data security and privacy, pseudonymised study identification 


\section{Box 1 Clinical endpoints}

Primary:

- Change in $\mathrm{HbA}_{1 \mathrm{C}}(\%)$ after 6 months between groups.

Secondary:

- Change in $\mathrm{HbA}_{1 \mathrm{C}}(\%)$ after 12 months between groups.

- Change in health literacy (HLS-EU-Q16, score) between 6 and 12 months between groups.

- Change in daily physical activity (IPAQ, score) after 6 and 12 months between groups.

- Change in average steps per days (7-day average of steps/day measured by pedometers) after 6 and 12 months between groups.

- Change in eating behaviour (FEV, score) after 6 and 12 months between groups.

- Change of quality of life (SF-36, score) after 6 and 12 months between groups.

- Change in healthcare costs $(€$, health claims data of health insurance fund) after 6 and 12 months between groups.

- Change in weight (kg) after 6 and 12 months.

- Change in waist circumference $(\mathrm{cm})$ after 6 and 12 months.

- Change in LDL-cholesterol concentrations (mg/dL) after 6 and 12 months.

- Change in HDL-cholesterol concentrations (mg/dL) after 6 and 12 months.

- Change in triglyceride concentration (mg/dL) after 6 and 12 months.

- Change in systolic blood pressure ( $\mathrm{mm} \mathrm{Hg}$ ) after 6 and 12 months.

- Change in diastolic blood pressure ( $\mathrm{mm} \mathrm{Hg}$ ) after 6 and 12 months.

- Number of the combined endpoint '4-point MACE' after 6 and 12 months.

FEV, questionnaire about eating behaviour; HbA1C, glycosylated haemoglobin; HDL, high density cholesterol; HLS-EU-Q16, Health Literacy Survey Questionnaire; IPAQ, International Physical Activity Questionnaire; LDL, Iow density cholesterol; MACE, major cardiovascular events defined as cardiovascular death, non-fatal stroke, non-fatal myocardial infarction, hospitalisation due to angina pectoris; SF-36, Short Form-36 Questionnaire.

codes (study IDs) as well as standardised email addresses are used to register in LS apps.

Furthermore, 7-day paper-based food diaries corresponding to these three points in time will be recorded by the study participants and evaluated by nutrition specialists at TUM.

For safety monitoring and analysis, adverse events will be obtained and immediately forwarded to the study site at TUM to be revaluated by a safety committee. All evaluations are performed according to SOPs to ensure objective evaluations and high data quality.

\section{Study endpoints}

The primary endpoint is a change in $\mathrm{HbA}_{1 \mathrm{C}}$ levels from baseline to 6 months of LS compared with UC (reduction of $\mathrm{HbA}_{1 \mathrm{C}}-0.4 \%$ from baseline level).

Secondary endpoints (box 1) include changes in health literacy, PA, eating behaviour, QoL, numerous cardiovascular risk factors, the number of major cardiovascular events (cardiovascular death, non-fatal stroke, non-fatal myocardial infarction, hospitalisation for angina pectoris and/or coronary revascularisation) and healthcare costs after 6 and 12 months. The self-reported questionnaires cover the following topics: HLS-EU-Q16 assessing health literacy, including items regarding disease management, prevention and health promotion ${ }^{15}$; IPAQ assessing PA during the last 7 days, including items for time spent on moderate, vigorous or sitting activities ${ }^{16}$; FEV assessing eating behaviour, including items regarding cognitive control of eating habits, restrained eating, disturbance of eating habits and experienced feelings of hunger ${ }^{17}$; and SF-36 assessing QoL, including items regarding physical and mental well-being. ${ }^{18}$

\section{Intervention}

The intervention is centrally provided by the study group at TUM, which means that the individual study sites can focus on patient recruitment and follow-up investigations. This also ensures a standardised and objective support of patients enrolled to the trial.

\section{Lifestyle intervention group (LS)}

Patients assigned to LS will receive individualised recommendations on nutrition as well as on their individual exercise programme. They will also receive twoweekly newsletters on PA, motivation and nutrition by mail.

\section{Exercise and daily activities}

The individual exercise training consists of both endurance and strength training and starts 2 weeks after randomisation. It is provided and monitored via the LeIKD app (IDS Diagnostic Systems AG, Ettlingen, Germany). The patients will be allocated to one of four different levels of fitness based on their maximum exercise capacity (percentage of normal peak oxygen consumption $\left(\% \dot{\mathrm{V}}{ }_{2} \text { peak }\right)^{19}$ and relative $\dot{\mathrm{V}} \mathrm{O}_{2}$ peak $\left.(\mathrm{mL} / \mathrm{min} / \mathrm{kg})\right)$, as further depicted in table 2 , at baseline and 6 months visit.

Despite different templates for each level, the initial aim is to encourage all patients to exercise on a daily basis. Every template consists of four endurance and two strength training sessions per week with an initial duration of 10-15 min and an increase of duration (up to 30-50 min) and intensity over time. The endurance training varies between low, moderate and vigorous intensities, as well as continuous and interval sessions. Intensities are individually calculated according to a threshold-based approach,

Table 2 Thresholds for endurance and strength exercise group allocations based on maximum exercise capacity

\begin{tabular}{|c|c|c|}
\hline $\begin{array}{l}\text { Exercise } \\
\text { training } \\
\text { level }\end{array}$ & $\begin{array}{l}\text { Thresholds for } \\
\text { endurance exercise } \\
\text { group allocation based } \\
\text { on percentage of } \\
\text { normal } \dot{\mathrm{V}}_{2} \text { peak }(\%)\end{array}$ & $\begin{array}{l}\text { Thresholds for } \\
\text { strength exercise } \\
\text { group allocation } \\
\text { based on relative } \dot{\mathrm{V}} \mathrm{O}_{2} \\
\text { peak }(\mathrm{mL} / \mathrm{min} / \mathrm{kg})\end{array}$ \\
\hline 1 & $\leq 75.0$ & $\leq 16.0$ \\
\hline 2 & $75.1-90.0$ & $16.1-22.0$ \\
\hline 3 & $90.1-110.0$ & $22.1-30.0$ \\
\hline 4 & $>110.0$ & $>30.0$ \\
\hline
\end{tabular}

$\dot{\mathrm{V}}{ }_{2}$ peak: peak oxygen uptake. 
and incorporate patients' CPET data in order to provide a best-practice individual exercise recommendation. ${ }^{20} 21$ All endurance sessions will be monitored by a heart rate sensor (H7 Heart rate sensor, Polar, Kempele, Finland) and recorded in the LeIKD app.

The strength training includes more than 30 different exercises with multiple variations and levels of intensity. All exercises can be performed at home and may incorporate conventional household items such as water bottles. Each session includes several exercises targeting different muscle groups (whole body, lower extremities, upper extremities, trunk muscles) and consists of at least two sets per exercise.

In addition to the LeIKD app, patients will receive a printed booklet with all exercises and access to an online platform, where they have access to instructional videos.

\section{Training adaptation}

The core laboratory for exercise training (TUM) will be able to access all exercise data via secured web platform to monitor adherence of the participants. In order to increase adherence and motivation, patients can choose their preferred mode of endurance training (eg, riding a bike or walking). Furthermore, patients are contacted via phone in weeks 3, 5, 9, 13, 17 and 21 (before t1) by exercise physiologists from TUM to review potential problems and, if necessary, individually adapt the type, amount, duration and/or intensity of the exercise sessions. Despite potential changes, the exercise training aims at increasing the duration to at least $150 \mathrm{~min}$ per week of moderate PA, or a mixture of moderate-to-vigorous PA with less than 150 min per week to meet general health recommendations.

Beyond structured endurance and strength training, patients are encouraged to increase their daily PA. Therefore, based on the average steps per day in the first 2 weeks, patients are advised to gradually increase their daily PA within 6 months. After 6 months (t1), heart rate zones for the different intensity domains will be adapted according to the results of CPET at 6 months visit. Two weeks later, all LS patients will be contacted by phone to be informed about their progress and the upcoming exercise schedule. The second part of the study primarily aims at maintaining the level of PA and exercise without regular feedback. Therefore, the patients will receive another exercise schedule based on their preferences (eg, less sessions with longer duration) in order to improve long-term motivation and adherence. No further individual contact is planned until the subsequent visit after 12 months (t2). In case of urgent safety-related issues or technical problems, the exercise programme will be individually adapted by study personnel if requested.

\section{Nutrition monitoring}

In addition to 7-day food diaries in weeks 1 (after t0), 27 (t1) and 52 (t2), patients in the LS will be asked to complete food diaries in weeks 5 and 14. Based on their food diaries, they will receive individualised nutritional advice based on the principle of energy density ${ }^{22}$ by either mail or local postal service centrally coordinated from nutritionists at TUM. This information will primarily contain recommendations about replacing high density (calories per gram) with lower density level. ${ }^{22}$ This principle is intended to lead to a reduction in caloric intake. ${ }^{22}$ Patients will be engaged to improve food choices based on principles of a balanced and healthy diet according to the German Nutrition Society (DGE). Nutritional advices focus on quality of food, consumption of fruits and vegetables, preferring calorie-free beverages and avoiding alcoholic drinks, preferably consuming rather fish than meat and avoid highly processed foods. This will empower the patient to make healthier, qualitatively better food choices based on their individual meal preferences and thereby support weight maintenance or reduction.

\section{Health literacy}

To increase health literacy particularly regarding PA and nutrition, all LS patients will receive two weekly emails with background information on PA, motivation and nutrition along with cooking recipes.

In order to improve health literacy, all patients are advised to measure their own blood glucose concentrations at different times during the day, as well as in different activity contexts, including (but not limited to) before and after one of their strength or endurance training sessions.

\section{Patient and public involvement}

Patients have not been involved in the development of the initial study protocol. Patients participate when adapting the individual exercise intervention and change of diet according to the nutritionist's feedback, see the Intervention section. A group of random patients was invited to participate at a focus group meeting to discuss and further develop questionnaires.

\section{Usual care}

Patients in the UC group are treated according to the ESC guidelines on the management of stable coronary artery disease $^{4}$ and all patients will receive detailed standard recommendations on nutrition and $\mathrm{PA}^{2324}$ after the baseline examination ( $\mathrm{t} 0$ ) by email. These include a recommendation to perform $150 \mathrm{~min}$ of moderate or $75 \mathrm{~min}$ of higher intensity activities and several other lifestylerelated advices (see online supplemental document) to assure even access of information for every patient. They will not receive a lifestyle intervention or individualised feedback.

\section{Claims data from statutory health insurance fund}

In order to investigate the effect of the intervention on health economics, a cost-effectiveness analysis based on claims data from one statutory health insurance (SHI) fund will be carried out as part of this study's health economic evaluation. Claims data can be obtained and analysed on an individual basis, as these data are directly 
transmitted from healthcare providers to SHI funds for billing and reimbursement purposes.

\section{Sample size calculation}

Based on the results of the ENHANCE trial ${ }^{6}$ (mean difference of $0.4 \% \pm 1.8 \%, 80 \%$ statistical power, $5 \%$ significance level and an estimated dropout rate of $15 \%$ ), a number of 750 patients have been calculated to detect a statistically significant difference of the primary endpoint between the groups. For an equally distributed comparison between patients living in urban and rural areas, the estimated number has been doubled to 1500 .

\section{Data handling}

The evaluation is based on three data sources: patient reported data, medical data and SHI claims data.

Patient-reported data include, among others, questionnaires (assessed and documented at study sites), PA data (eg, pedometer by app) and nutrition diaries (centrally coordinated at TUM). Medical data are either assessed and documented by study site. SHI claims data are derived by health insurance. All data sources are pseudonymised in the same way by study IDs. No interim analysis will be conducted.

Patient-reported data and medical data will be extracted from the electronic data capture system secuTrial by the interActive Systems GmbH (Berlin, Germany). secuTrial is compliant with the Directive 2001/20/EC guidelines for Good Clinical Practice. Moreover, the compliance with all legal requirements for the interActive Systems $\mathrm{GmbH}$ in the handling of all collected data will be monitored by the evaluating institute within the scope of order data processing. SHI claims data will be provided by the stationary health insurance fund TK. The different sources are linked by study ID and validated for the analyses.

\section{Statistical analysis}

The aim of the statistical analysis is to compare changes in outcomes between different points in time (ie, baseline, after 6 months and after 12 months) and study groups (ie, LS and UC). Therefore, outcomes will be measured and analysed at all different points in time in both study groups (see figure 1) following the intention-to-treat principle. Statistical analysis has been described in a study evaluation protocol. Descriptive variables and changes in outcomes will be summarised separately for both study groups. An analysis of covariance will be conducted to evaluate all between-subject effects and to identify interactions of between-subject and within-subject effects, including within-covariate interactions. To consider relevant differences between the study groups regarding the baseline variables, adjustments can be made for possible confounders (eg, comorbidities). Additionally, an analysis of variance will be used to evaluate all within-subject effects between the three points in time. To compare differences between groups, t-tests (for parametric data) or Mann-Whitney U tests (for non-parametric data) will be applied. Additionally, multivariate analyses are performed to identify and investigate factors influencing the study's primary and secondary outcomes. Sensitivity analyses will be conducted to assess the robustness of the results to variation in key parameters. This will comprise per-protocol analyses and, if applicable, as-treated analyses, which will be defined in a statistical analysis plan before the database is locked.

In order to investigate our intervention's effect on health economics, a cost-effectiveness analysis will be carried out as part of the health economic evaluation. In this analysis, a modified societal perspective will be adopted to account for healthcare system costs. This perspective will cover the costs of our intervention, as well as the general costs for medical care (eg, outpatient and inpatient diagnostics and treatment), pharmaceuticals, rehabilitation, remedies and aids, as well as sick leave payments during the study period. Lastly, the incremental cost-effectiveness ratio will be calculated as the change in average costs between the IC and CG divided by the difference in average change on $\mathrm{HbA}_{1 c}$ levels between the LS and UC. All analyses will be performed with a significance level of $\alpha=0.05$ and will not be adjusted for multiplicity. Therefore, analyses of secondary endpoints should be interpreted as hypothesis-generating.

\section{ETHICS AND DISSEMINATION}

The LeIKD study has been approved by the local ethics committee of the TUM (protocol 27.03.2018, identification code: $144 / 18$-S) as well as each ethics committee responsible for other participating study sites. The study will be conducted according to the World Medical Association Declaration of Helsinki. Protocol modifications must be approved by all legal authorities if necessary and further communicated. Study participation is voluntary and patients are only included after written and verbal informed consent. Patients can withdraw from study participation at any time without personal legal consequences. The results of the study will be published by the study group in presentations, articles and reports, independently of the funding sponsor. The authors were involved in study proposal ( $\mathrm{MH}, \mathrm{VA}, \mathrm{BH})$, are part of the steering committee (SN, VA, BH, MH and SM) and will be involved in study coordination and dissemination (all authors).

\section{Discussion}

The LeIKD study will be one of the largest randomised trials investigating a telemedical lifestyle intervention in patients suffering from both CIHD and T2DM. This multimorbid, mostly elderly patient population has a significantly increased morbidity and mortality and poses a substantial and increasing socioeconomic burden. ${ }^{2}$

In the UK Prospective Diabetes Study (UKPDS), a $1 \%$ reduction in $\mathrm{HbA}_{1 \mathrm{C}}$ was associated with a significant $(\mathrm{p}<0.001)$ reduced risk in diabetes-related deaths $(-21 \%)$, myocardial infarction $(-14 \%)$ and microvascular 
complications $(-37 \%) .{ }^{25}$ However, the authors state that they were not able to define a threshold regarding diabetic complications and that any improvement of diabetic control by reduction of $\mathrm{HbA}_{1 \mathrm{C}}$, independent of baseline $\mathrm{HbA}_{1 \mathrm{C}}$ concentration, reduces the risk of complication. ${ }^{25}$ The European Medicines Agency has set their non-inferiority margin for changes in $\mathrm{HbA}_{1 \mathrm{c}}$ to $0.3 \%,{ }^{26}$ which is slightly less than what we expect as a treatmentrelated mean difference between the groups $(-0.4 \%)$ based on the results of the ENHANCE trial. ${ }^{6}$

Currently, it has not been investigated before in a large randomised trial, whether QoL and health economic aspects may significantly be improved by a telemedical lifestyle intervention for patients with cardiovascular disease. ${ }^{11}$ Therefore, the LeIKD study will address an important and challenging patient population, will closely evaluate adherence to lifestyle interventions, will include a telemedicine platform and devices, and will include economic data analysis.

The TEMA-HF 1 study including 160 patients with HFrEF showed promising results by telemedical intervention (data of weight, blood pressure and heart rate electronically submitted to hospital, alert if necessary) during 6 months of rehabilitation after acute heart failure. ${ }^{27}$ Results for all-cause mortality $(17.5 \%$ death rate in the control group vs $5 \%$ in the intervention group, $\mathrm{p}=0.012$ ), hospitalisation $(0.82 \pm 0.93$ hospitalisations/subject in the control group vs $0.80 \pm 0.97$ in the intervention group, $\mathrm{p}=0.934)$ and hospitalisation costs $(€ 1458 \pm 3420$ in the control group vs $€ 902 \pm 2277$ in the intervention group, $\mathrm{p}=0.23$ ) are promising, even though the sample size was relatively small. ${ }^{27}$

The long-term implementation of lifestyle intervention is a key challenge in all lifestyle trials. In the HF ACTION study in HFrEF, the authors identified (1) low motivation, (2) limitations due to comorbidities and (3) the disease condition itself as the main contributors towards low adherence rates. ${ }^{9}$ The LeIKD study aims at changing the patients' lifestyle in their personal environment, an approach that has succeeded previously. ${ }^{12}$ Moreover, as monotonous exercise regimens will most likely lead to a decline in adherence, the LeIKD study will deliberately extend variation of exercise regarding volume, intensity, load of training, individualised training scheme and target to improve exercise capacity.

Next to the individual feedback, we intensively aim at increasing health literacy (by using pedometers, blood glucose monitoring and weekly newsletters). Health literacy and the role of personal guidance are thought to play a major role in patients' long-term adherence. This will be quantified within the LeIKD study by health literacy questionnaires and comparison of episodes with individual feedback for the first 6 months and thereafter without.

In LeIKD, individual nutritional recommendations aim at motivating the patients to change their diet by triggering their intrinsic motivation rather than imposing strict rules from the study personnel. Patients design their own diet supported by general recommendations from newsletters, as well as implementing feedback from nutritional specialists by replacing food of high by low density. Thereby, we will follow an educational approach to support the lifestyle changes and improve health literacy.

SHI claims data from the trial will add valuable information regarding the economic impact of a lifestyle intervention. Furthermore, it will provide an overview of current treatment quality in Germany in light of the observed conditions. Compared with clinical trials, claims data analyses have been shown to reflect real-life healthcare provisions. ${ }^{28}$ Other benefits of this data source include cost-efficient data generation and access to a large study population. Linking all data will ideally provide a deeper insight into real-life treatment and patient-relevant assessments, thereby also contributing to improve healthcare research.

The LeIKD study represents one of the largest lifestyle intervention trials in the field of cardiology and diabetology. Furthermore, to our knowledge, no other trial has thus far evaluated the effects of a 12-month telemedical lifestyle intervention in a population with both CIHD and T2DM. The multicentre approach as well as the close link to one of the largest insurance companies in Germany, which identifies patients according to their ICD codes in the area of study centres, will ensure an optimised recruitment strategy and potential for optimal health economic analyses and long-term follow-up. The LeIKD study will thereby contribute towards establishing a standard procedure for a telemedical approach of lifestyle intervention in a population with CIHD and T2DM.

\section{Author affiliations}

${ }^{1}$ Department of Prevention and Sports Medicine, Technical University of Munich, Munchen, Germany

${ }^{2}$ DZHK (German Centre for Cardiovascular Research), partner site Munich, Munich Heart Alliance, Munich, Germany

${ }^{3}$ Institute for Applied Health Services Research GmbH (INAV), Berlin, Germany

${ }^{4}$ Department of Health Economics and Health Policy, Hannover Medical School, Hannover, Germany

${ }^{5}$ Techniker Krankenkasse, Hamburg, Germany

${ }^{6}$ IDS Diagnostic Systems GmbH, Bonn, Germany

Acknowledgements We deeply thank Alessandra Boscheri, MD, Munich, Germany, for significantly contributing to the grant application and Felix Gass for his significant impact for study coordination.

Contributors PvK, HS, SN, AD, AM, SD, BH, MW, JS, VA, SM and MH contributed to the conception and/ or design and/or planned analysis of the work. PvK drafted the manuscript with contribution from SM, HS, AM, SN and MH. All authors critically revised the manuscript and gave final approval.

Funding The study is funded by the Federal Joint Committee (Innovationsfonds des Gemeinsamen Bundesausschuss G-BA, www.innovationsfonds.g-ba.de), reference number 01NVF17015, which has no impact on data collection, analysis, interpretation or scientific dissemination. The Federal Joint Committee is a national funding organisation sponsoring healthcare research.

Competing interests All other authors report no conflict of interest despite the grant from Federal Joint Committee (Innovationsfonds des Gemeinsamen Bundesausschuss G-BA), from null, during the conduct of the study. MH reports institutional funding from Techniker Krankenkasse. Health Insurance Company, Hamburg, Germany, outside the submitted work. BH reports personal fees from IDS Diagnostic Systems, outside the submitted work.

Patient consent for publication Not required. 
Provenance and peer review Not commissioned; externally peer reviewed.

Supplemental material This content has been supplied by the author(s). It has not been vetted by BMJ Publishing Group Limited (BMJ) and may not have been peer-reviewed. Any opinions or recommendations discussed are solely those of the author(s) and are not endorsed by BMJ. BMJ disclaims all liability and responsibility arising from any reliance placed on the content. Where the content includes any translated material, BMJ does not warrant the accuracy and reliability of the translations (including but not limited to local regulations, clinical guidelines, terminology, drug names and drug dosages), and is not responsible for any error and/or omissions arising from translation and adaptation or otherwise.

Open access This is an open access article distributed in accordance with the Creative Commons Attribution Non Commercial (CC BY-NC 4.0) license, which permits others to distribute, remix, adapt, build upon this work non-commercially, and license their derivative works on different terms, provided the original work is properly cited, appropriate credit is given, any changes made indicated, and the use is non-commercial. See: http://creativecommons.org/licenses/by-nc/4.0/.

\section{ORCID iD}

Pia von Korn http://orcid.org/0000-0003-4528-2681

\section{REFERENCES}

1 , Rydén L, Grant PJ, et al, Authors/Task Force Members. ESC guidelines on diabetes, pre-diabetes, and cardiovascular diseases developed in collaboration with the EASD: the task force on diabetes, pre-diabetes, and cardiovascular diseases of the European Society of cardiology (ESC) and developed in collaboration with the European association for the study of diabetes (EASD). Eur Heart $J$ 2013;34:3035-87.

2 Kotseva K, Wood D, De Bacquer D, et al. EUROASPIRE IV: a European Society of cardiology survey on the lifestyle, risk factor and therapeutic management of coronary patients from 24 European countries. Eur J Prev Cardiol 2016;23:636-48.

3 , Sarwar N, Gao P, et al, Emerging Risk Factors Collaboration. Diabetes mellitus, fasting blood glucose concentration, and risk of vascular disease: a collaborative meta-analysis of 102 prospective studies. Lancet 2010;375:2215-22.

4 , Montalescot G, Sechtem U, et al, Task Force Members. 2013 ESC guidelines on the management of stable coronary artery disease: the task force on the management of stable coronary artery disease of the European Society of cardiology. Eur Heart J 2013;34:2949-3003.

5 The Look AHEAD Research Group. Cardiovascular effects of intensive lifestyle intervention in type 2 diabetes. $N$ Engl J Med Overseas Ed 2013;369:145-54.

6 Sevick MA, Korytkowski M, Stone RA, et al. Biophysiologic outcomes of the enhancing adherence in type 2 diabetes (enhance) trial. J Acad Nutr Diet 2012;112:1147-57.

7 Lean ME, Leslie WS, Barnes AC, et al. Primary care-led weight management for remission of type 2 diabetes (DiRECT): an openlabel, cluster-randomised trial. Lancet 2018;391:541-51.

8 Stewart RAH, Held C, Hadziosmanovic N, et al. Physical activity and mortality in patients with stable coronary heart disease. J Am Coll Cardiol 2017;70:1689-700.

9 O'Connor CM, Whellan DJ, Lee KL, et al. Efficacy and safety of exercise training in patients with chronic heart failure: HF-ACTION randomized controlled trial. JAMA 2009;301:1439-50.

10 World Health Organization. Telemedicine: opportunities and developments in member states: report on the second global survey on eHealth. Global Observatory for eHealth Series 2010;2.
11 Zhu Y, Gu X, Xu C. Effectiveness of telemedicine systems for adults with heart failure: a meta-analysis of randomized controlled trials. Heart Failure Rev 2019.

12 Koehler F, Koehler K, Deckwart O, et al. Efficacy of telemedical interventional management in patients with heart failure (TIM-HF2): a randomised, controlled, parallel-group, unmasked trial. Lancet 2018;392:1047-57.

13 Chan A-W, Tetzlaff JM, Altman DG, et al. SPIRIT 2013 statement: defining standard protocol items for clinical trials. Ann Intern Med 2013;158:200-7.

14 Guazzi M, Arena R, Halle M, et al. 2016 focused update: clinical recommendations for cardiopulmonary exercise testing data assessment in specific patient populations. Circulation 2016;133:e694-711.

15 Jordan S, Hoebel J. Gesundheitskompetenz von Erwachsenen in Deutschland. Bundesgesundheitsblatt Gesundheitsforschung. Gesundheitsschutz 2015;58:942-50.

16 Taheri S, Zaghloul H, Chagoury O, et al. Effect of intensive lifestyle intervention on bodyweight and glycaemia in early type 2 diabetes (DIADEM-I): an open-label, parallel-group, randomised controlled trial. Lancet Diabetes Endocrinol 2020;8:477-89.

17 Pudel V, Westenhoefer J. Fragebogen zum Essverhalten: Handanweisung. Goettingen, 1989.

18 Kato A, Fujimaki Y, Fujimori S, et al. Psychological and behavioural patterns of stigma among patients with type 2 diabetes: a crosssectional study. BMJ Open 2017;7:e013425.

19 Gläser S, Ittermann T, Schäper C, et al. [The Study of Health in Pomerania (SHIP) reference values for cardiopulmonary exercise testing]. Pneumologie 2013;67:58-63.

20 Mezzani A, Hamm LF, Jones AM, et al. Aerobic exercise intensity assessment and prescription in cardiac rehabilitation: a joint position statement of the European association for cardiovascular prevention and rehabilitation, the American association of cardiovascular and pulmonary rehabilitation and the Canadian association of cardiac rehabilitation. Eur J Prev Cardiol 2013;20:442-67.

21 Pescatello L, Arena R, Riebe D. ACSM's guidelines for exercise testing and prescription. J Can Chiropr Assoc 2014;58:328.

22 Pérez-Escamilla R, Obbagy JE, Altman JM, et al. Dietary energy density and body weight in adults and children: a systematic review. $J$ Acad Nutr Diet 2012;112:671-84.

23 Bundesärztekammer (BÄK), Kassenärztliche Bundesvereinigung (KBV), Arbeitsgemeinschaft der Wissenschaftlichen Medizinischen Fachgesellschaften (AWMF). PatientenLeitlinie zur Nationalen VersorgungsLeitlinie "Therapie des Typ-2-Diabetes" (National care guideline for the treatment of type 2 diabetes). 1, 2015.

24 Bundesärztekammer. BÄK), Kassenärztliche Bundesvereinigung (KBV), Arbeitsgemeinschaft Der Wissenschaftlichen Medizinischen Fachgesellschaften (AWMF). Chronische Koronare Herzkrankheit: KHK. Patientenleitlinie zur Nationalen VersorgungsLeitlinie 2017;1.

25 Stratton IM, Adler Al, Neil HA, et al. Association of glycaemia with macrovascular and microvascular complications of type 2 diabetes (UKPDS 35): prospective observational study. BMJ 2000;321:405-12.

26 European Medicines Agency. Guideline on clinical investigation of medicinal products in the treatment or prevention of diabetes mellitus, 2018.

27 Dendale P, De Keulenaer G, Troisfontaines P, et al. Effect of a telemonitoring-facilitated collaboration between general practitioner and heart failure clinic on mortality and rehospitalization rates in severe heart failure: the TEMA-HF 1 (TElemonitoring in the management of heart failure) study. Eur J Heart Fail 2012;14:333-40.

28 Neubauer S, Kreis K, Klora M, et al. Access, use, and challenges of claims data analyses in Germany. Eur J Health Econ 2017;18:533-6. 